\title{
Gonads and strife: Sex hormones vary according to sexual orientation for women and stress indices for both sexes
} \author{
Jens C. Pruessner ${ }^{\mathrm{b}, \mathrm{g}, \mathrm{h}}$, Sonia J. Lupien ${ }^{\mathrm{e}}$ \\ a Department of Psychiatry, Columbia University, New York, NY, United States \\ ${ }^{b}$ Department of Neurology and Neurosurgery, McGill University, Montreal, Quebec, Canada \\ ${ }^{\mathrm{c}}$ Department of Psychology, Concordia University, Montreal, Quebec, Canada \\ d Départment de Neuroscience, Université de Montréal, Montreal, Quebec, Canada \\ e Départment de Psychiatrie, Université de Montréal, Montreal, Quebec, Canada \\ ${ }^{\mathrm{f}}$ Department of Psychology, Bishop's University, Sherbrooke, Quebec, Canada \\ ${ }^{g}$ Department of Psychiatry, McGill University, Montreal, Quebec, Canada \\ h Department of Psychology, McGill University, Montreal, Quebec, Canada
}

Robert-Paul Juster ${ }^{\mathrm{a}, *}$, Daniel Almeida ${ }^{\mathrm{b}}$, Christopher Cardoso $^{\mathrm{c}}$, Catherine Raymond $^{\mathrm{d}}$, Philip Jai Johnson ${ }^{a}$, James G. Pfaus ${ }^{c}$, Adrianna Mendrek ${ }^{\mathrm{e}, \mathrm{f}}$, Annie Duchesne ${ }^{\mathrm{f}}$,

Keywords:

Sexual orientation

Testosterone

Estradiol/progesterone

Dehydroepiandrosterone-sulphate

Cortisol

Allostatic load

\begin{abstract}
A B S T R A C T
This study assessed sexual orientation and psychobiological stress indices in relation to salivary sex hormones as part of a well-validated laboratory-based stress paradigm. Participants included 87 healthy adults that were on average 25 years old who self-identified as lesbian/bisexual women $(n=20)$, heterosexual women $(n=21)$, gay/bisexual men $(n=26)$, and heterosexual men $(n=20)$. Two saliva samples were collected fifteen minutes before and fifteen minutes after exposure to a modified Trier Social Stress Test to determine testosterone, estradiol, and progesterone concentrations via enzyme-immune assaying. Mean sex hormones were further tested in association to stress indices related to cortisol systemic output (area under the curve with respect to ground) based on ten measures throughout the two-hour visit, allostatic load indexed using 21 biomarkers, and perceived stress assessed using a well-validated questionnaire. Results revealed that lesbian/bisexual women had higher overall testosterone and progesterone concentrations than heterosexual women, while no differences were found among gay/bisexual men in comparison to heterosexual men. Lesbian/bisexual women and heterosexual men showed positive associations between mean estradiol concentrations and allostatic load, while gay/bisexual men and heterosexual women showed positive associations between mean testosterone and cortisol systemic output. In summary, sex hormone variations appear to vary according to sexual orientation among women,

but also as a function of cortisol systemic output, allostatic load, and perceived stress for both sexes.
\end{abstract}

\section{Introduction}

Exploration into the neurobiological correlates of sexual orientation has had a controversial history. Early research reflected homosexuality's classification as a mental illness until 1973, at which time the American Psychiatric Association removed it from its diagnostic manual (Friedman and Downey, 1994). Biological

\footnotetext{
* Corresponding author at: Columbia University, Dept. of Psychiatry, Division of Behavioral Medicine and Division of Gender, Sexuality, and Health, Columbia University Medical Center, 622 West 168th St., PH1540G, New York, NY 10032, United States.

E-mail address: rjuster2108@cumc.columbia.edu (R.-P. Juster).
}

explanations of non-heterosexual behavior have often hypothesized a dysregulation of sex-specific hormone profiles, resulting in anomalies in the organizational and activational effects of these hormones on the neurodevelopment of circuitry underlying species-specific sexual behavior. Animal models involving prenatal androgen deficits, for example, were first believed to cause male homosexuality (Phoenix et al., 1959), while prenatal androgen overabundance presumably resulted in female homosexuality. Our study endeavours to show that such sex hormone differences assumed to be attributable to sexual orientation are also modulated by unexplored stress phenomena.

Advances in behavioral neuroscience has led to the introduction of sophisticated genetic models involving non-functional androgen receptors, further allowing researchers to characterize the relation- 
ship between androgens, the masculinization of the rodent brain, and sexual behavior (Zuloaga et al., 2008). Guided by this literature, perturbations in the human prenatal environment during a critical period (between 10 to 22 weeks gestation) might expose the human fetus to androgen imbalances that have the potential to alter structure and function of key neuroanatomical regions implicated in human sexuality (Ellis and Ames, 1987). While this early exposure of androgens to the developing brain is understood to affect post-natal sex/gender, sexual orientation, and related behaviors, the literature has been inconclusive and thus reflects the multifactorial nature of human sexuality.

Several human studies during the 1970s using analytes extracted from either urine, serum, or plasma revealed that men belonging to a sexual minority showed higher testosterone (Brodie et al., 1974; Doerr et al., 1976; Tourney and Hatfield, 1973), lower testosterone (Brodie et al., 1974; Kolodny et al., 1972; Kolodny et al., 1971; Loraine et al., 1971; Loraine et al., 1970; Pillard et al., 1974; Rohde et al., 1977; Stahl et al., 1976), no differences in testosterone (Barlow et al., 1974; Birk et al., 1973; Doerr et al., 1973; Jaffee et al., 1980), higher estrogen (Doerr et al., 1973; Doerr et al., 1976), or lower estrogen (Evans, 1972) when compared to heterosexual controls. Likewise, among sexual minority women compared to age-matched heterosexual controls, studies have reported lower estrogen (Loraine et al., 1971; Loraine et al., 1970), higher testosterone (Loraine et al., 1971; Loraine et al., 1970), no differences in testosterone (Downey et al., 1987), and no differences in estrogen or progesterone (Griffiths et al., 1974; Seyler et al., 1978). Similarly for gonadotropins, studies reporting elevated luteinizing hormone concentrations among sexual minority men (Kolodny et al., 1972) and women (Loraine et al., 1971) have been matched by an abundance of research failing to show differences in luteinizing hormone, follicle stimulating hormone, as well as prolactin (Friedman and Frantz, 1977; Jaffee et al., 1980; Kolodny et al., 1971; Parks et al., 1974).

In a critical review of the literature, Meyer-Bahlburg concluded that findings were overall inconsistent among sexual minority men (Meyer-Bahlburg, 1977). By contrast, about one-third of participating sexual minority women manifested elevated androgen levels while otherwise showing no endocrine abnormalities (MeyerBahlburg, 1979). Importantly, methodological differences between studies rendered comparisons and any final conclusions difficult. This body of human research did not support the neurohormonal hypothesis of sexual orientation (Banks and Gartrell, 1995). MeyerBahlberg acknowledged that some early researchers were rightfully cautious in their conclusions (Meyer-Bahlburg, 1977). In particular, the inconsistencies in observed HPG-axis patterns might not be the primary cause of sexual minority orientation, but rather a secondary consequence related to unmeasured factors such as psychosocial stress (Kolodny et al., 1972; Meyer-Bahlburg, 1979).

Stress researchers during the 1970s began showing that psychological factors could modulate the HPG-axis. For example, a longitudinal study among military men undergoing stressful training revealed that plasma testosterone levels were lowest during the earlier novice phase compared to the later senior phase (Kreuz et al., 1972). This paralleled the pioneering work of Mason who systematically studied stressful situations (e.g., parachute jumping, air-traffic controlling) and identified key psychological determinants (e.g., novelty, uncontrollability) that activated stress responses (Mason, 1968). Unfortunately this knowledge was not applied to understanding the mixed HPG-axis literature on sexual orientation, despite speculation that psychosocial stress might be involved in study inconsistencies. Because sexual minorities are at an increased risk for stress-related pathologies due to stigma and discrimination (IOM, 2011; Meyer, 2003), it is highly probable that unique psychosocial contexts influence HPG-axis profiles and might confound the literature describing biological differences focusing purely on sexual orientation, identity, \& behavior.

Advances in psychosocial and biological approaches to studying stress propelled an entire field of psychoneuroendocrine research aimed at identifying mechanisms of disease susceptibility. In particular, the development of laboratory-based stress induction paradigms have substantiated that the sexes differ in their stress response patterns of the stress hormone cortisol easily collected via saliva. Studies using the popular Trier Social Stress Test or TSST (Kirschbaum et al., 1993) consistently show that men mount a greater cortisol response than women of reproductive age (Kirschbaum et al., 1992). In turn, women show further attenuation when using oral contraceptives (Kirschbaum et al., 1995) or during the high estrogen (follicular) phase of their menstrual cycles as opposed to during the luteal phase (Kirschbaum et al., 1999).

Beyond sex differences in stress reactivity, research applying stress biomarkers are beginning to be used to understand how stigma affects the health and wellbeing of sexual minorities (Hatzenbuehler et al., 2013). Our group has recently provided novel evidence that sexual orientation modulates cortisol reactivity. Specifically, lesbian/bisexual women show higher post-stressor cortisol concentrations compared to heterosexual women, while gay/men show overall lower cortisol concentrations compared to heterosexual men after controlling for basal sex hormone concentrations (Juster et al., 2015). In addition to this, we found that sexual minority participants who had disclosed their sexual orientation to family and friends evidenced lower morning cortisol levels and less psychiatric symptoms than those who had not completely disclosed irrespective of sex (Juster et al., 2013b). These reports suggest that important psychosocial and behavioral factors may result in distinct biological signatures.

It remains unknown however, how circulating sex hormones in the context of the TSST paradigm vary as a function of one's sexual orientation, and how these associations are further modulated by stress phenomena. In the current study, we explored whether sexual minorities differ from same-sex heterosexual controls in terms of salivary testosterone, estradiol, and progesterone concentrations before and after exposure to the TSST. Guided by our previous reports showing that biopsychosocial stress is uniquely experienced between and within sexual orientations (Juster et al., 2015; Juster et al., 2016a; Juster et al., 2013b), we further assessed whether changes in sex hormones were associated with cortisol systemic output summarized using 10 measurements throughout the TSST, allostatic load indexed using 21 stress-related biomarkers, and finally perceived stress.

Given the mixed findings in the HPG-axis literature on sexual orientation and the lack of studies linked to stress indices, we did not hypothesize directionality of associations. We did, however, hypothesize that psychobiological stress indices would correlate with mean sex hormone concentrations beyond those associations attributable to sexual orientation.

\section{Methods}

\subsection{Participants}

Eighty-seven participants ages $18-45(M=24.61 \pm 0.61 S E)$ identifying as lesbian or gay ( 8 women and 20 men), bisexual (13 women and 6 men), and heterosexual (20 women and 21 men) were recruited from Montreal as part of a broader research program (Juster et al., 2015; Juster et al., 2016a; Juster et al., 2013b). To equalize groups due to fewer lesbians and bisexual men, we combined lesbian/gay with bisexual individuals (20 women and 26 men) and contrasted them to heterosexuals (20 women and 21 men). 
Table 1

Sample descriptive statistics according to sex and sexual orientation.

\begin{tabular}{|c|c|c|c|c|c|c|}
\hline Information & Sample & Lesbian/Bisexual + & Heterosexual $q$ & Gay/Bisexual $\sigma^{7}$ & Heterosexual $\sigma^{7}$ & $p$ \\
\hline$N$ & 87 & 20 & 20 & 26 & 21 & - \\
\hline \multicolumn{7}{|l|}{ Demographic: } \\
\hline Age, $M(S E)$ & $24.61(0.61)$ & $24.10(1.34)$ & $25.45(1.13)$ & $23.77(0.98)$ & $25.33(1.47)$ & 0.685 \\
\hline \multicolumn{7}{|l|}{ Race/ethnicity: } \\
\hline White, \% & 70.1 & 75.0 & 55.0 & 73.1 & 76.2 & 0.147 \\
\hline Black, \% & 5.7 & 15.0 & 10.0 & 0.0 & 0.0 & 0.147 \\
\hline Asian, \% & 12.6 & 0.0 & 10.0 & 23.1 & 14.3 & 0.147 \\
\hline Hispanic, \% & 6.9 & 0.0 & 20.0 & 3.8 & 4.8 & 0.147 \\
\hline Arab, \% & 4.6 & 10.0 & 5.0 & 0.0 & 4.8 & 0.147 \\
\hline \multicolumn{7}{|l|}{ Occupation: } \\
\hline Workers, \% & 34.5 & 40.0 & 35.0 & 23.1 & 42.9 & 0.490 \\
\hline Students, \% & 65.5 & 60.0 & 65.0 & 76.9 & 57.1 & 0.490 \\
\hline Working/studying hours/week, $M(S E)$ & $28.02(1.82)$ & $28.40(3.63)$ & $28.5(4.38)$ & $29.96(3.24)$ & $24.91(3.54)$ & 0.789 \\
\hline \multicolumn{7}{|l|}{ Sexual orientation ${ }^{A}$ : } \\
\hline Sexual attractions, $M(S E)$ & $3.51(0.26)$ & $4.75(0.33)$ & $1.40(0.15)$ & $5.96(0.30)$ & $1.29(0.10)$ & $<0.001$ \\
\hline Sexual behaviors, $M(S E)$ & $3.34(0.26$ & $4.25(0.44)$ & $1.30(0.11)$ & $6.00(0.29)$ & $1.114(0.8)$ & $<0.001$ \\
\hline Sexual fantasies, $M(S E)$ & $3.61(0.26)$ & $5.15(0.25)$ & $1.65(0.25)$ & $5.81(0.32)$ & $1.29(0.12)$ & $<0.001$ \\
\hline Lifestyle preferences, $M(S E)$ & $3.37(0.27)$ & $5.15(0.44)$ & $1.115(0.08)$ & $5.54(0.33)$ & $1.10(0.07)$ & $<0.001$ \\
\hline Sexual identity, $M(S E)$ & $3.52(0.27)$ & $5.05(0.43)$ & $1.15(0.08)$ & $6.04(0.26)$ & $1.19(0.09)$ & $<0.001$ \\
\hline \multicolumn{7}{|l|}{ Socio-economic: } \\
\hline Post-secondary education, \% & 95.3 & 95.0 & 90.0 & 100.0 & 95.0 & 0.575 \\
\hline Personal annual income, \$ CAD, $M(S E)$ & $16,000(0.17)$ & $14,500(0.34)$ & $19,000(0.53)$ & $14,000(0.19)$ & $16,800(0.33)$ & 0.737 \\
\hline Household annual income, \$ CAD, $M(S E)$ & $32,100(0.32)$ & $37,000(0.68)$ & $25,000(0.54)$ & $27,100(0.52)$ & $39,000(0.75)$ & 0.311 \\
\hline \multicolumn{7}{|l|}{ Health and well-being: } \\
\hline Medication use, \% & 18.4 & 25.0 & 15.0 & 15.4 & 14.3 & 0.105 \\
\hline Oral contraceptive use, $\%$ & 16.1 & 20.0 & 50.0 & - & - & 0.017 \\
\hline Minor physical condition, \% & 34.5 & 50.0 & 40.0 & 23.1 & 28.6 & 0.238 \\
\hline \multicolumn{7}{|l|}{ Psychiatric history: } \\
\hline None, \% & 28.7 & 15.0 & 40.0 & 26.9 & 33.3 & 0.522 \\
\hline Past history, \% & 8.0 & 15.0 & 5.0 & 11.5 & 33.3 & 0.522 \\
\hline Family history, \% & 35.6 & 35.0 & 45.0 & 30.8 & 33.3 & 0.522 \\
\hline Both past and family history, \% & 27.6 & 35.0 & 10.0 & 30.8 & 33.3 & 0.522 \\
\hline \multicolumn{7}{|l|}{ Subjective dimensions ${ }^{B}$ : } \\
\hline Self-rated health, $M(S E)$ & $3.72(0.09)$ & $3.50(0.21)$ & $3.95(0.14)$ & $3.69(0.17)$ & $3.75(0.20)$ & 0.419 \\
\hline Self-rated physique, $M(S E)$ & $3.37(0.10)$ & $3.15(0.22)$ & $3.70(0.18)$ & $3.27(0.14)$ & $3.40(0.28)$ & 0.284 \\
\hline Self-rated diet, $M(S E)$ & $3.30(0.11)$ & $3.25(0.19)$ & $3.40(0.25)$ & $3.34(0.19)$ & $3.15(0.25)$ & 0.832 \\
\hline \multicolumn{7}{|l|}{ Behavioral: } \\
\hline \multicolumn{7}{|l|}{ Tobacco smoking: } \\
\hline Smokers, \% & 11.5 & 5.0 & 10.0 & 11.5 & 19.0 & 0.376 \\
\hline Social smokers, \% & 14.9 & 10.0 & 5.0 & 19.2 & 23.8 & 0.376 \\
\hline Non-smokers, \% & 73.6 & 85.0 & 85.0 & 69.2 & 57.1 & 0.376 \\
\hline \multicolumn{7}{|l|}{ Alcohol consumption (weekly): } \\
\hline 0 or infrequently, \% & 25.3 & 25.0 & 50.0 & 18.1 & 4.8 & 0.134 \\
\hline 1 to $5, \%$ & 40.2 & 45.0 & 40.0 & 26.9 & 52.4 & 0.134 \\
\hline 6 to $10, \%$ & 26.4 & 25.0 & 10.0 & 38.5 & 28.6 & 0.134 \\
\hline 11 or more, $\%$ & 8.0 & 5.0 & 0.0 & 11.5 & 14.3 & 0.134 \\
\hline \multicolumn{7}{|l|}{ Elicit drug use: } \\
\hline None, \% & 66.7 & 60.0 & 85.0 & 61.5 & 61.9 & 0.334 \\
\hline Occasional (monthly or annually), \% & 24.1 & 35.0 & 5.0 & 30.8 & 23.8 & 0.334 \\
\hline Regular (daily or weekly), \% & 9.2 & 5.0 & 10.0 & 7.7 & 14.3 & 0.334 \\
\hline \multicolumn{7}{|l|}{ Interpersonal: } \\
\hline Single, $\%$ & 72.1 & 60.0 & 80.0 & 80.8 & 65.0 & 0.315 \\
\hline Children, \% & 4.7 & 10.0 & 5.0 & 0.0 & 5.0 & 0.463 \\
\hline Siblings, \% & 86.0 & 95.0 & 85.0 & 80.8 & 85.0 & 0.578 \\
\hline Parents alive, \% & 94.2 & 95.0 & 90.0 & 92.3 & 100.0 & 0.559 \\
\hline Infrequent family gatherings, $\%$ & 36.1 & 30.0 & 30.0 & 57.7 & 20.0 & 0.169 \\
\hline Non-religious/spiritual, \% & 78.8 & 76.5 & 84.2 & 84.0 & 68.4 & 0.569 \\
\hline
\end{tabular}

During screening, women provided information concerning their menstrual cycles (e.g., date of last menses, average cycle days) and oral contraceptive use. One woman who did not provide information on reproductive functioning and was therefore removed from main analyses. The main exclusionary criteria in this study were major health problems, severe mental illness, or use of synthetic glucocorticoids. Transgender individuals were not solicited due to hormonal treatments that directly affect the HPG-axis; however, this represents a group that ought to be included in future research given critical health inequalities (IOM, 2011) The sample's information on demographics, socio-economics, health, wellbeing, lifestyle behaviors, and interpersonal features are reported in Table 1.

\subsection{General protocol}

This study was approved by the research ethics board of the Institut universitaire en santé mentale de Montréal. Upon a 15-min study explanation and screening interview via telephone, eligible participants were scheduled for a first visit at the Centre for Studies on Human Stress. All participants provided informed consent upon arrival to our laboratory. Testing was conducted between July 2010 to November 2010, constraining any seasonal variation that confound sex hormones (van Anders et al., 2006).

A morning visit was scheduled between 8:00AM and 11:00AM and lasted approximately $40 \mathrm{~min}$. This visit involved a blood draw from a certified nurse, a Continental breakfast to break a $12 \mathrm{~h}$ fast, completion of questionnaires, and finally, detailed instructions for 
take-home biomarker collection protocols first reported elsewhere (Juster et al., 2013b). Between visits spaced on average one week apart, participants completed take-home materials before returning to the laboratory.

An afternoon/evening visit was scheduled between 12:00PM and 7:00PM and lasted $120 \mathrm{~min}$. The current study focuses on this afternoon/evening visit in which participants were exposed to a modified version of the TSST while providing saliva samples. Upon completion of study requirements, participants were explained their blood results in detail and compensated with \$50 CAD upon debriefing.

\subsection{Sexual orientation}

Sexual orientation was ascertained and cross-validated using three combined methods: (1) response to one of three separate advertisements searching for either lesbian/gay, bisexual, or heterosexual participants; (2) asking participants their identified sexual orientation in an open-ended manner during telephone screening; and (3) administration of a modified 5-item Klein Sexual Orientation Scale (Klein et al., 1990). This instrument uses a 7-point Likert scale from 1 (other sex only) to 7 (same sex only) to assess sexual attractions, sexual behaviors, sexual fantasies, lifestyle preference, and sexual identity. The entire sample's responses were internally consistent ( $\alpha=0.982$ ). Based on correspondence among these three methods, sexual orientation was coded as "sexual minority" $(n=46)$ or "heterosexual" $(n=41)$.

\subsection{Perceived distress}

The 14-item Perceived Stress Scale (Cohen et al., 1983) measures perceived stress using a 5 -point Likert scale from 0 (never) to 4 (very often). Original psychometric properties revealed strong internal consistency (mean $\alpha=0.85$ ), test-retest reliability (mean $r=0.85$ ), and evidence of concurrent validity with depression (mean $r=0.71$ ) and physical complaints (mean $r=0.59$ ) among young students. In the current sample, perceived stress showed strong internal consistency $(\alpha=0.88)$.

\subsection{Stress reactivity paradigm}

During the two-hour afternoon visit to our laboratory, participants were exposed to a modified version (Andrews et al., 2007; Wadiwalla et al., 2010) of the Trier Social Stress Test or TSST (Kirschbaum et al., 1993). Upon a ten-minute anticipation phase, participants were led to a separate room where they were asked to deliver a five-minute mock job interview followed by five minutes of mental arithmetic in front of an unseen, ostensible behavioral expert seated behind a one-way mirror. The participant and the "behavioral expert" communicated via an intercommunication device and the participant's performance was recorded by a video camera. A seminal meta-analysis (Dickerson and Kemeny, 2004) posits that laboratory-based stressors eliciting social-evaluative threat include evaluative audiences, negative social comparison(s), and/or recorded performance that maximize HPA-axis reactivity.

Previous studies by our group demonstrate that placing the evaluative audience behind a one-way mirror ('panel-out') further maximizes between-sex differences in cortisol stress reactivity. Specifically, men exposed to this type of the TSST show no significant differences in HPA-axis reactivity when compared to the standard performance in front of the audience ('panel-in'); (Andrews et al., 2007). In contrast, heterosexual women exposed to the 'panel-out' condition show decreased cortisol stress reactivity in comparison to heterosexual women in the 'panel-in' condition (Wadiwalla et al., 2010). Our recent study employing heterosexual women in the 'panel-out' condition also reports comparable decreased cortisol reactivity (Marin et al., 2012); however, it is unknown how this is related to sex hormone variations.

\subsection{Visit order}

Participants visited our laboratory twice: (1) morning visit for a blood draw to assess allostatic load and (2) afternoon visit for the TSST. Visit order was counterbalanced randomly to manipulate experienced novelty of the testing environment. In the first group (morning/afternoon; $n=49$ ), participants received a blood draw in the morning during their first visit and were exposed to the TSST in the afternoon during their second visit about a week later; this was reversed for the second group (afternoon/morning; $n=37$ ). Because the second group was arriving for the first time to our laboratory when exposed to the TSST, we expected that they would be more distressed than the first group who had already familiarized themselves with the setting. This acclimation is believed to help diminish participants' distress experienced in novel testing environments (Sindi et al., 2013)

While this did not significantly modulate cortisol concentrations in a previous report (Juster et al., 2015), preliminary analyses were conducted in the current study to assess whether this manipulation modulated sex hormone dynamics. The HPG-axis is believed to be involved in anticipatory states and expectation of preoperative stress (Gerra et al., 2000). Additionally noteworthy in terms of daytime variability, it has been suggested that testosterone collection should be conducted in the afternoon and evening to provide the best possible representation of an individual's general responsiveness to social interactions and not those based on natural diurnal variation (Gray et al., 2004).

\subsection{Saliva collection and endocrine assays}

Two saliva samples (approximately $2 \mathrm{~mL}$ per sample) were collected -15 min pre-TSST and +15 min post-TSST to assess salivary testosterone, estradiol, and progesterone at each time-point. Collection was achieved using the passive drool method guided with sterilized straws. Samples were frozen immediately upon collection at $-20^{\circ} \mathrm{C}$ and assays were performed within three months of testing to prevent degradation known to influence HPG-axis biomarkers (Granger et al., 2004). Because collection occurred between 12:00PM and 7:00PM ( $M=2: 34 \mathrm{PM}, S E=0.11)$, we assessed for circadian variation in sex hormone values for the entire sample: no significant correlations with arrival time were found. Thirty minutes prior to saliva sampling, participants were instructed to avoid major meals, cigarettes, caffeinated/sugary beverages, and dairy products. They were furthermore asked to refrain from oral hygiene and strenuous physical activity two hours before sampling.

Ten saliva samples destined for cortisol determination were collected in ten-minute intervals at the following time-points: $-40 \mathrm{~min},-30 \mathrm{~min},-20 \mathrm{~min},-10 \mathrm{~min}$ (anticipation phase), and immediately before the TSST, as well as $+10 \mathrm{~min},+20 \mathrm{~min},+30 \mathrm{~min}$, $+40 \mathrm{~min}$, and $+50 \mathrm{~min}$ thereafter. At the end of the testing sessions, these saliva samples were frozen at $-20^{\circ}$ until assaying. A previous report using this sample showed that lesbian/bisexual had higher cortisol concentrations than heterosexual women $+40 \mathrm{~min}$ post-TSST, while gay/bisexual men showed lower overall cortisol concentrations than heterosexual men throughout the session (Juster et al., 2015). In the current analysis, we summarize all 10 cortisol time-points to assess associations with mean sex hormone concentrations.

All saliva samples were analyzed at the Centre for Studies on Human Stress (www.humanstress.ca). Prior to assaying each biomarker, frozen samples were brought to room temperature to be centrifuged at $1500 \mathrm{xg}$ ( $3000 \mathrm{rpm}$ ) for $15 \mathrm{~min}$. For testosterone determination, we used an expanded range enzyme immune assay 
kit (Salimetrics ${ }^{\circledR}$, State College, PA, Catalogue No. 1-2402) where the lower limit of sensitivity is $1 \mathrm{pg} / \mathrm{ml}$. For estradiol, or more specifically $17 \beta$-estradiol, determination, we used a high sensitivity enzyme immune assay kit (Salimetrics ${ }^{\circledR}$, State College, PA, Catalogue No. 1-3702) where the range of detection is $1-32 \mathrm{pg} / \mathrm{ml}$. For progesterone determination, we used a high sensitivity enzyme immune assay kit (Salimetrics ${ }^{\circledR}$, State College, PA, Catalogue No. 11502 ) where the lower limit of sensitivity is $5 \mathrm{pg} / \mathrm{ml}$. Inter-assay and intra-assay coefficients of variance were respectively below $12.80 \%$ and $6.34 \%$ for testosterone, $3.7 \%$ and $6.78 \%$ for estradiol, and $8.08 \%$ and $6.78 \%$ for progesterone. For cortisol determination, we used a high sensitivity enzyme immune assay kit (Salimetrics ${ }^{\circledR}$ State College, PA, Catalogue No. 1-3102) where the range of detection is between $0.012-3 \mathrm{ug} / \mathrm{dL}$. Assays were run in duplicates and averages were used in statistical analyses.

\subsection{Allostatic load biomarkers}

Allostatic load is defined as the multi-systemic 'wear and tear' that chronic stress and unhealthy behaviors exact on the body and brain (McEwen and Stellar, 1993). Twenty-one biomarkers were used to calculate a count-based allostatic load index constructed according to the sample's distribution for high-risk percentiles as previously done (Seeman et al., 1997). Systemic biomarkers represent neuroendocrine (salivary cortisol was transformed into both AM cortisol and PM cortisol slopes to capture dynamic diurnal HPA-axis fluctuations; serum dehydroepiandrosterone-sulphate; $12 \mathrm{~h}$ overnight urinary adrenalin, noradrenalin, and dopamine), immune/inflammatory (plasma interleukin-6 and tumour-necrosis factor-alpha; serum c-reactive protein; and serum fibrinogen preserved with sodium citrate), metabolic (serum albumin, creatinine, insulin, glycosylated haemoglobin (\%), total cholesterol, highdensity lipoprotein, and triglycerides; waist-to-hip ratio measured with graduated tape and calculated by dividing respective inches; body mass index calculated as mass $(\mathrm{kg})$ divided by height $\left(\mathrm{m}^{2}\right)$; and cardiovascular (mean of three seated systolic and diastolic blood pressure recordings measured with an electronic sphygmomanometer; A\&D Medical@: Model UA-631 V) functioning. Table 2 lists all biomarkers used with their respective cut-offs and group characterizations.

Previous allostatic load findings in this sample showed that gay/bisexual men had lower allostatic load levels than heterosexual men and that this was driven by lower triglycerides, body mass indices, and trending tumour-necrosis factor-alpha concentrations (Juster et al., 2013a). Among sexual minorities, those that enacted avoidance coping strategies during sexual identity formation and disclosure evidenced elevated allostatic load and psychosocial distress (Juster et al., 2016a). In the current analysis, our rationale for using allostatic load was to assess potential convergence of associations with salivary sex hormones and to assess differences between women for individual biomarkers which we did not previously assess since women did not show allostatic load differences.

\subsection{Statistical analysis}

All statistical analyses were run using the Statistical Package for the Social Science Version 22 for Macintosh. Group differences in descriptive information are reported in Table 1. Groups only differed according to sexuality as expected (all ps<0.001), while heterosexual women used oral contraceptives more than lesbian/bisexual women $(p=0.017)$.

Due to the vast inter-individual differences in sex hormone concentrations and intra-individual variations as a function of contextual stimuli and reproductive considerations, we carefully accounted for potential confounders in our main analyses

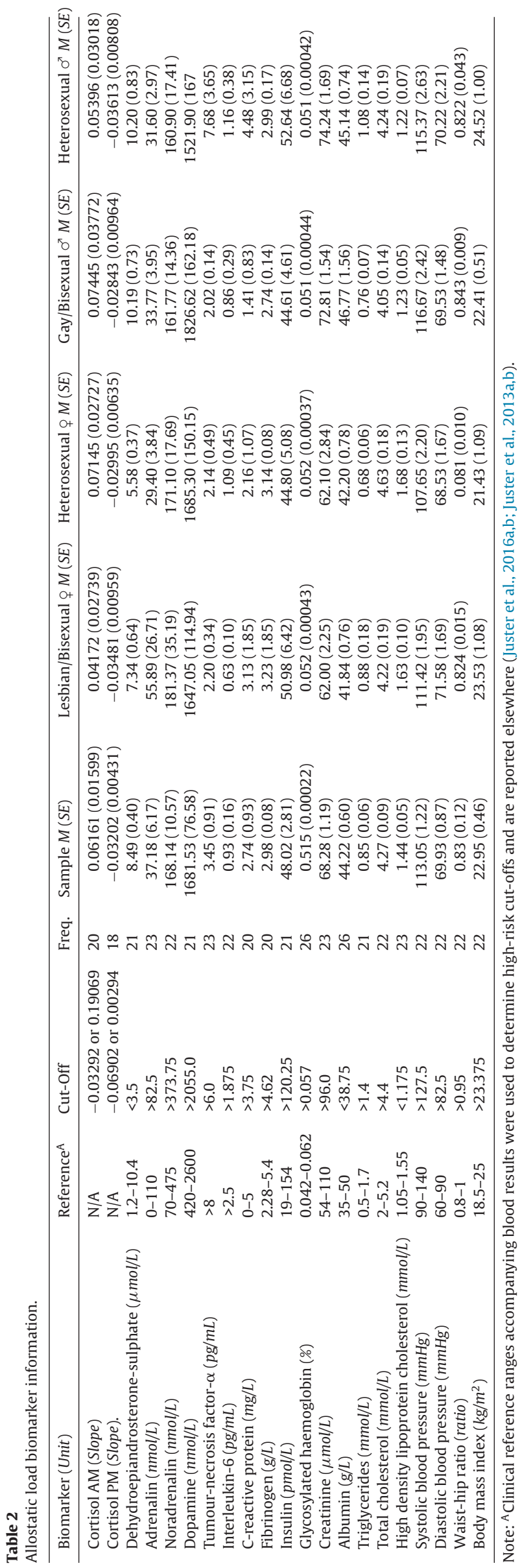


of repeated measures. Specifically for each sex hormone in sequence, potential confounding effects were first assessed using repeated-measures ANOVAs as a function of (1) visit order (morning/afternoon: $n=49$; afternoon/morning group: $n=37$ ), (2) menstrual cycle divided equally according to follicular lesbian/bisexual women $(n=11)$ and heterosexual women $(n=11)$ as well as luteal lesbian/bisexual women $(n=9)$ and heterosexual women ( $n=9$ ), and finally (3) oral contraceptive use (users: $n=14$; non-users: $n=26$ ) that was more common among heterosexual women (users: $n=10$, non-users: $n=10$ ) than lesbian/bisexual women (users: $n=4$; non-users: $n=16$ ). Using information that contrasted the TSST visit date and the date of last menstruation for women $(M=18.25, S E=2.01)$, preliminary one-way ANOVAs of study outcomes as a function of menstrual cycle status revealed no significant differences between women in the follicular (days 1-14) and luteal (days 15-28) phases. Note that menstrual status was unknown for one participant, so final sample $N=86$.

Mixed-design repeated-measures analysis of covariance (ANCOVA) was run with sexual orientation entered as the between-subjects factor and sex hormones (two measures of testosterone, estradiol, and progesterone each $15 \mathrm{~min}$ before and $15 \mathrm{~min}$ after TSST exposure) entered as the within-subject factors in sex-specific analyses while controlling for age and visit order as well as menstrual cycle status and oral contraceptive use among women. Post-hoc analyses employed one-way ANOVA or paired-sample $t$-test as required. In all such analyses, effect sizes are reported throughout and can be interpreted according to the following conventions: $\eta^{2} \cong 0.01$ represents a small effect, $\eta^{2}{ }_{P} \cong 0.06$ a medium effect, and $\eta^{2}{ }_{P} \cong 0.14$ constitute a large effect (Fritz et al., 2012).

Next, we assessed associations among mean sex hormones in relation to cortisol systemic output, allostatic load, and perceived stress using bivariate correlations split according to sex. Time-dependent stress reactive cortisol concentrations were transformed into summary scores based on the area under the curve formulae based on the trapezoid formula (Pruessner et al., 2003). The area under the curve with respect to ground (AUCg) was calculated to represent systemic output throughout the TSST visit.

In supplemental analyses, we followed with an exploration of 21 individual biomarker differences using ANCOVAs controlling for age and bivariate correlations with other study variables.

\section{Results}

\subsection{Testosterone}

In preliminary analyses of visit order, the afternoon/morning group showed higher salivary testosterone concentrations among both women $\left(\mathrm{F}_{(1,38)}=7.003, \quad p=0.012, \eta^{2}{ }_{P}=0.156\right)$ and men $\left(\mathrm{F}_{(1,44)}=7.645, p=0.008, \eta^{2}=0.148\right)$ in comparison to the morning/afternoon group. A time effect was also found among men $\left(F_{(1,44)}=12.241, p<0.001, \eta^{2}{ }_{P}=0.218\right)$, revealing that testosterone concentrations significantly decreased following the TSST. Among women, menstrual status was not related to differences in concentrations of testosterone $(p=0.26)$. By contrast, oral contraceptive use was related to lower testosterone concentrations in comparison to non-use $\left(\mathrm{F}_{(1.38)}=6.267, p=0.017, \eta^{2}{ }_{P}=0.142\right)$.

For women (Fig. 1A), lesbian/bisexual women showed higher testosterone concentrations than heterosexual women $\left(F_{(1,34)}=8.648, p=0.006, \eta^{2}{ }_{P}=0.203\right)$. Covariation effects were found for visit order $\left(\mathrm{F}_{(1,34)}=4.182, p=0.049, \eta^{2}{ }^{2}=0.110\right)$ while trending for oral contraceptive use $\left(\mathrm{F}_{(1,34)}=3.591, p=0.067\right.$, $\left.\eta_{P}^{2}=0.096\right)$ and time $\mathrm{X}$ menstrual cycle phase $\left(\mathrm{F}_{(1,34)}=3.044\right.$ $p=0.090, \eta_{P}^{2}=0.082$ ).
For men (Fig. 1B), no significant within-subjects effects or interactions were detected ( $p s>0.364)$. Between-subjects results revealed no group differences ( $p s>0.15$ ) other than the covarying effect of visit order $\left(\mathrm{F}_{(1,42)}=4.892, p=0.032, \eta^{2}{ }_{P}=0.104\right)$.

\subsection{Estradiol}

Preliminary analyses demonstrated that visit order was not significantly related to salivary estradiol concentrations among women $(p=0.120)$ and men $(p=0.121)$ despite a trend towards a time by group interaction among women $\left(\mathrm{F}_{(1,38)}=2.991, p=0.092\right.$, $\left.\eta^{2}{ }_{p}=0.073\right)$ and a trend towards a time effect in men $\left(\mathrm{F}_{(1,44)}=3.411\right.$, $\left.p=0.071, \eta^{2}{ }_{P}=0.072\right)$ marked in both cases by non-significant decreases in response to the TSST. Estradiol concentrations did not differ according to menstrual cycle $(p=0.508)$ or according to oral contraceptive use $(p=0.233)$ among women.

For women (Fig. 1C), no between-subjects effects $(p s>0.41)$ or within-subjects effects or interactions ( $p s>0.18$ ) were detected for estradiol concentrations with the exception of a visit order covariation effect $\left(\mathrm{F}_{(1,34)}=4.881, p=0.034, \eta^{2}{ }_{P}=0.126\right)$.

For men (Fig. 1D), no between-subjects effects ( $p s>0.183$ ) or within-subjects effects or interactions $(p s>0.319)$ were detected for estradiol concentrations.

\subsection{Progesterone}

In preliminary analyses, the afternoon/morning group showed higher salivary progesterone concentrations than the morning/afternoon group among women $\left(F_{(1.38)}=4.083, p=0.050\right.$, $\left.\eta^{2}{ }^{2}=0.097\right)$ but not men $(p=0.272)$. For women, no group differences in progesterone concentrations were detected as a function of menstrual status ( $p=0.776)$ or oral contraceptives use $(p=0.282)$.

For women (Fig. 1E), lesbian/bisexual women displayed higher progesterone concentrations than heterosexual women $\left(\mathrm{F}_{(1,34)}=4.084, p=0.05, \eta^{2}{ }_{P}=0.107\right)$. Beyond a time X menstrual status covariation interaction $\left(\mathrm{F}_{(1,34)}=3.772, p=0.06, \eta^{2}{ }_{P}=0.10\right)$. No other between-subjects effects ( $p s>0.17$ ) or within-subjects effects or interactions ( $p s>0.13$ ) were detected.

For men (Fig. 1F), no significant findings were detected for between-subject effects ( $p s>0.224$ ) or within-subject effects or interactions $(p s>0.388)$.

\subsection{Sex-specific associations among mean sex hormones and stress indices}

Table 3 reports all descriptive statistics and correlative statistics for mean sex hormones, cortisol systemic output, allostatic load, and perceived stress stratified according to sex. First, testosterone was positively associated with cortisol systemic output for both sexes and with perceived stress for men. Second, estradiol was positively associated with allostatic load and perceived stress only among men. Third, progesterone was positively associated with cortisol systemic output for both sexes.

Re-analyses split further by sex and sexual orientation were next performed. For lesbian/bisexual women, allostatic load was positively associated with estadiol $(r=0.458, p=0.049)$. For heterosexual women, cortisol systemic output was positively associated with testosterone $(r=0.629, p=0.001)$ and progesterone $(r=0.483$, $p=0.012$ ). For gay/bisexual men, cortisol systemic output was positively associated with testosterone $(r=0.501, p=0.024)$. For heterosexual men, allostatic load was positively associated with estradiol $(r=0.508, p=0.022)$ and perceived stress was positively associated with testosterone $(r=0.676, p=0.001)$ and estradiol $(r=0.457, p=0.043)$. 
A

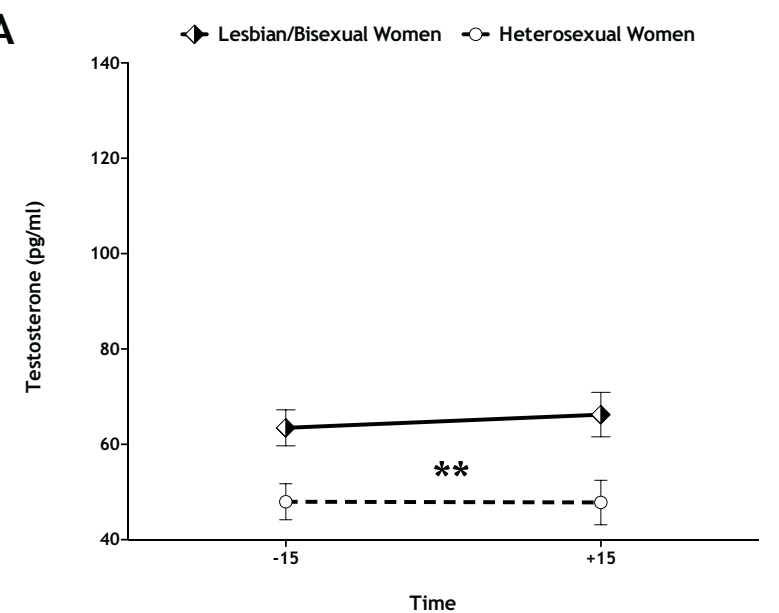

C

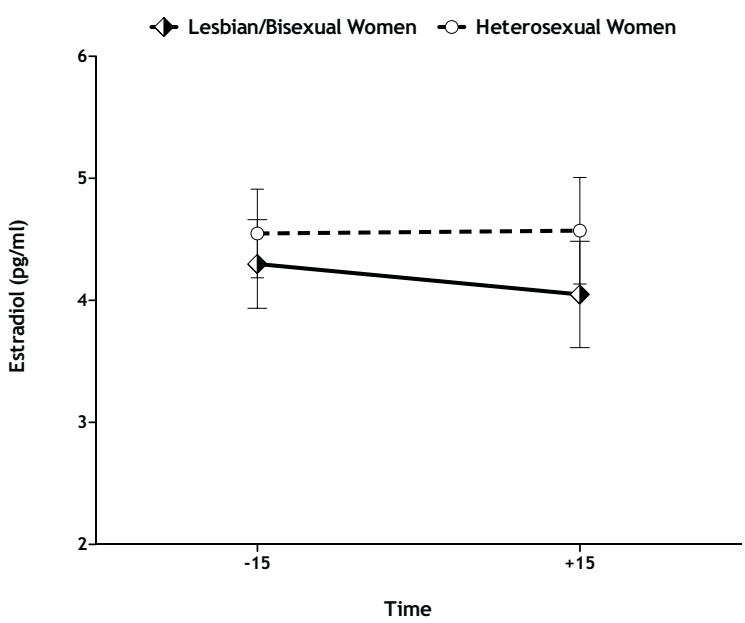

E

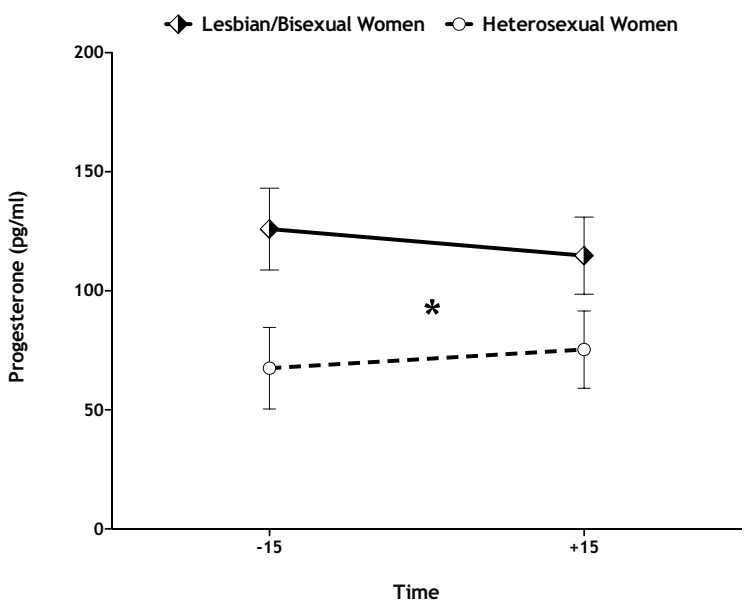

B

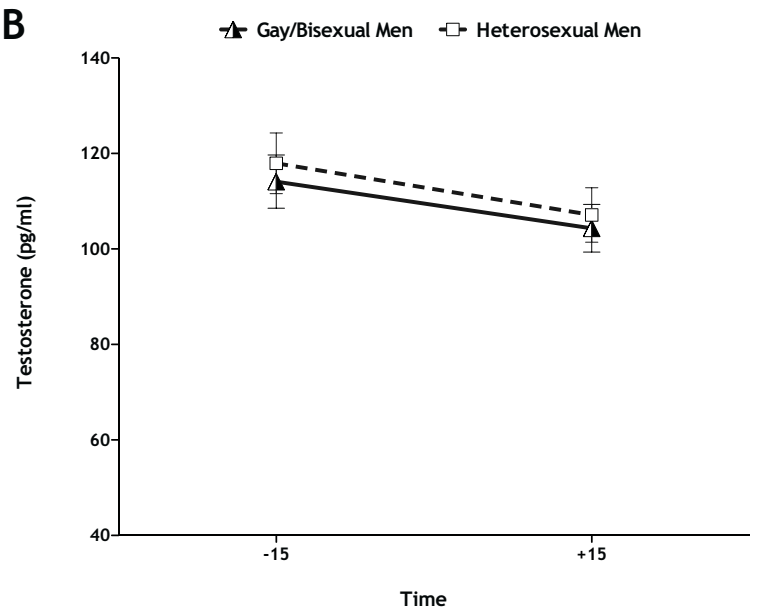

D

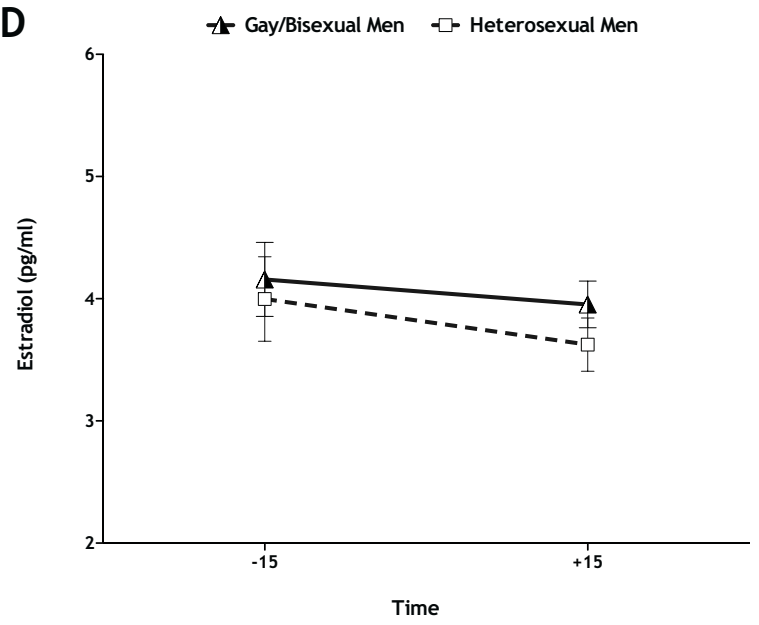

F

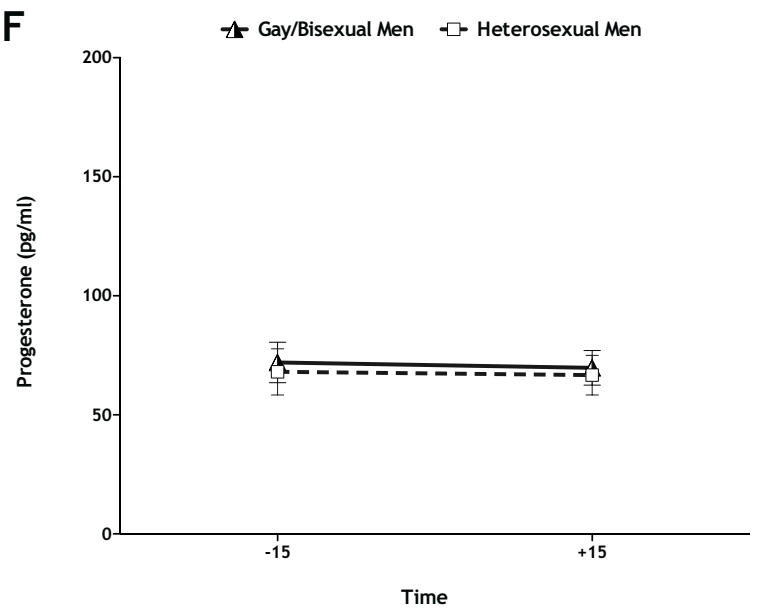

Fig. 1. Sex-specific estimated mean $( \pm$ SE) salivary testosterone (A and B), estradiol ( $C$ and $D)$, and progesterone $(E$ and $F)-15$ min before and +15 min after exposure to the Trier Social Stress Test. Values are adjusted for age and visit order for both sexes as well as menstrual status and oral contraception for women. Note: ${ }^{* *}=p<0.01 ;{ }^{*}=p<0.05$. 


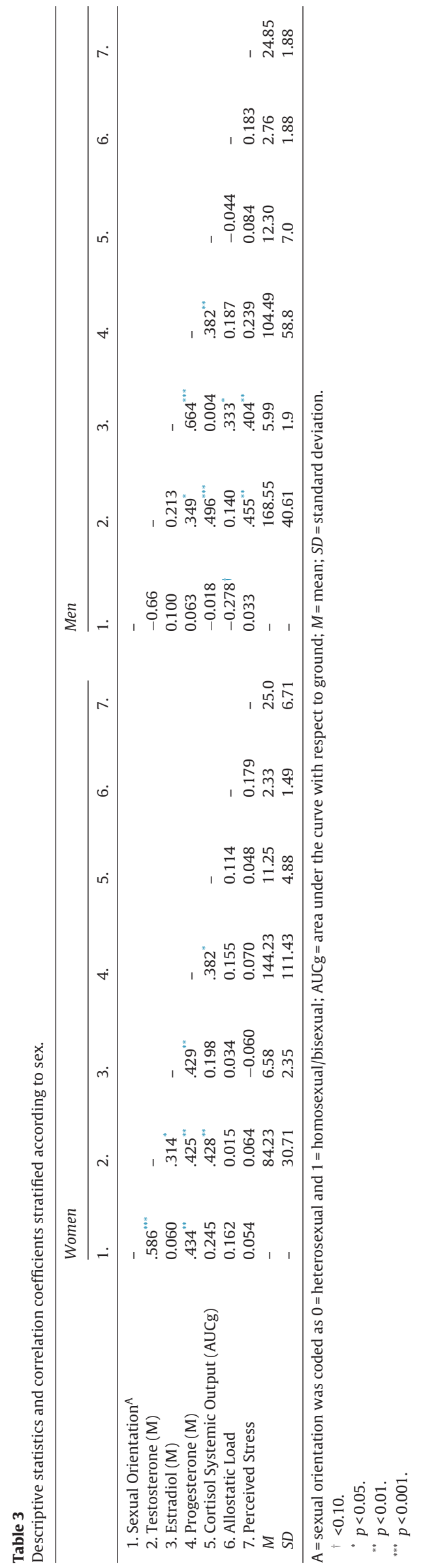

\subsection{Supplemental results for individuals biomarkers among women}

Individual biomarkers comprising the allostatic load index were assessed to explore convergence of salivary sex hormone findings reported above with other biomarkers extracted from blood, urine, etc. Analysis employed separate ANCOVAs controlling for age among women only. Results revealed that lesbian/bisexual women had higher levels of dehydroepiandrosterone-sulphate $\left(F_{(1.36)}=5.31, p=0.027, \eta^{2}=0.129\right.$; Fig. $\left.2 \mathrm{~A}\right)$ and lower levels of LDL-cholesterol $\left(\mathrm{F}_{(1,36)}=4.155, p=0.049, \eta^{2}{ }_{P}=0.103\right.$; Fig. 2 B $)$ than heterosexual women.

\section{Discussion}

This study explored whether unbound salivary testosterone, estradiol, and progesterone concentrations vary as a function of sexual orientation as well as by a comprehensive array of stress indices. We found that lesbian/bisexual women showed higher overall testosterone and progesterone concentrations as a group in comparison to heterosexual women, while men showed no differences in HPG-axis functioning. In supplemental analyses, lesbian/bisexual women also showed higher concentrations of the androgen precursor DHEA-S and lower LDL-cholesterol than heterosexual women. Besides these sexual orientation effects among women vis-à-vis basal biomarkers, we also found that mean sex hormones were associated to stress indices; namely, cortisol systemic output, allostatic load comprising 21 biomarkers, and perceived stress. Furthermore, our findings suggest that recalibrations in HPG-axis physiology may be explained by intertwined psychosocial processes (e.g., perceived stress), concomitant biobehavioral activities (e.g., cortisol dynamics), and cumulative physiological dysregulations (e.g., allostatic load) rather than strictly according to sexual orientation.

Independent of sex and sexual orientation, mean sex hormones varied according to stress indices. First, cortisol systemic output representing 10 repeated measurements throughout the TSST visit was positively associated with mean testosterone and progesterone levels for women and men. Second, we find novel evidence that allostatic load indexed with 21 biomarkers was correlated with estradiol only among men. Third, perceived stress was positively associated with testosterone and estradiol only among men. When broken down according to sex and sexual orientation groups, lesbian/bisexual women and heterosexual men showed similar positive associations between testosterone and cortisol (AUCg), while gay/bisexual men and heterosexual women showed positive associations between estradiol and AL. This intriguing "gender reversal" in directional associations is consistent with our cortisol reactivity findings in this sample whereby lesbian/bisexual women hyper-reacted like heterosexual men while gay/bisexual men hypo-reacted akin to heterosexual women (Juster et al., 2015).

Our findings among women contribute to a mixed literature. Specifically, higher basal testosterone concentrations in lesbian/bisexual women is in accord with some existing reports (Loraine et al., 1971; Loraine et al., 1970), but also in disaccord with studies showing no differences (Downey et al., 1987; Griffiths et al., 1974). Lesbian/bisexual women also demonstrated higher basal progesterone concentrations in comparison to heterosexual women. While this result is novel for women belonging to a sexual minority group, a study of 92 heterosexual women (Fleischman et al., 2015) found that homoerotic motivation was significantly and positively associated with salivary progesterone levels. It is important to note, however, that the variation in progesterone concentrations varied considerably. In addition, we also found for the first time that lesbian/bisexual women showed elevated 


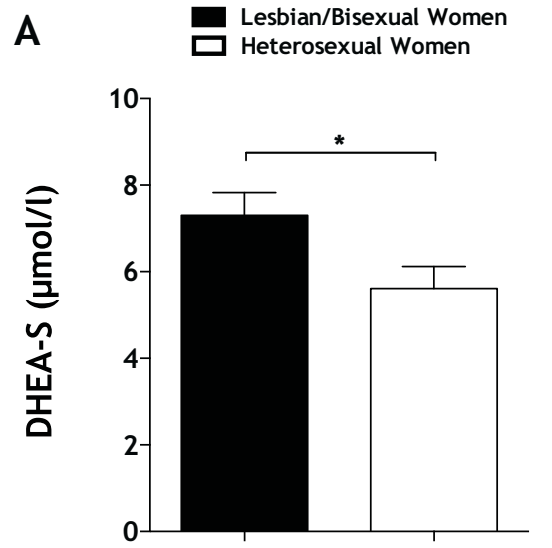

Sexual Orientation

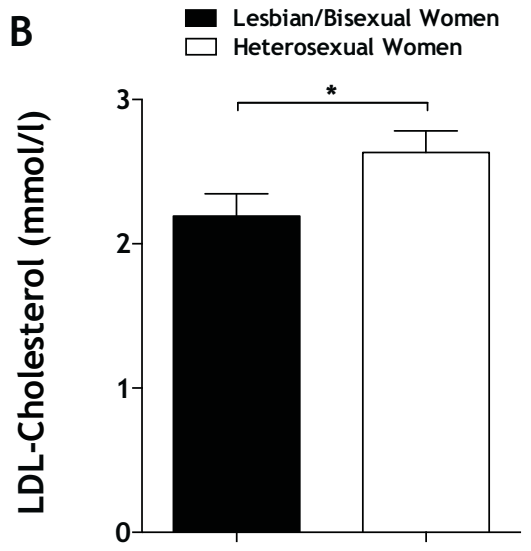

Sexual Orientation

Fig. 2. Women-specific estimated mean $( \pm$ SE) (A) dehydroepiandrosterone-sulphate (DHEA-S) and (B) low-density-lipoprotein (LDL)-cholesterol between lesbian/bisexual women and heterosexual women.

Note: ${ }^{*}=p<0.05$

concentrations of serum DHEA-S and reduced concentrations of LDL-cholesterol as part of blood draw on a separate day from the TSST session. This provides some convergence of evidence with salivary steroid hormone data that strengthens our conclusions. In addition, DHEA-S was positively correlated with testosterone, which is an expected direction of association given the biochemical synthesis of testosterone from DHEA-S through androstenedione.

A longstanding proposal in neuroendocrinology has been that elevated androgens among sexual minority women might be explained by environmental stress (Meyer-Bahlburg, 1979), and this may also be true of DHEA-S. Similar to cortisol, serum concentrations of DHEA-S are elevated in response to acute psychosocial stress induced by the TSST (Lennartsson et al., 2012b). Given that we also found that higher post-stressor cortisol reactivity in lesbian/bisexual women in comparison to heterosexual women (Juster et al., 2015), as well as the antagonizing relationship between DHEA-S and cortisol, it is possible that the unique psychosocial contexts driving this endocrine phenomenon may subsequently raise basal DHEA-S in a compensatory fashion. Finally, the synthesis of steroid hormones depends in part on LDLcholesterol, which explains why levels were low among sexual minority women.

Our findings among lesbian/bisexual women converge with literature suggesting a higher exposure to elevated prenatal androgens otherwise not present among men. For example, prenatal androgen exposure acts to reduce otoacoustic emissions (OAE; echo-like waveforms emitted by the cochlea) (McFadden, 2002) and to decrease the 2D:4D finger ratio (Manning, 2002). A meta-analysis showed that heterosexual women have a greater (more feminine) 2D:4D ratio than lesbian women, but no differences emerge for men (Grimbos et al., 2010). Lesbian/bisexual women also show decreased OAEs (McFadden and Pasanen, 1998). Furthermore, prenatal exposure to exogenous estrogens (e.g., diethylstilbestrol) in women is associated with non-heterosexual proclivities (Ehrhardt et al., 1985; Meyer-Bahlburg et al., 1995), but inconsistently among men (Hines, 2011). In the context of prenatal stress, one proposal why these sex hormone associations are not present in men may be due to testicular androgen production that compensates for stress alterations in androgen secretion that is absent among women (Hines, 2011). While prenatal conditions alone likely cannot determine sexual orientation (Ehrhardt and Meyer-Bahlburg, 1981), the higher androgens among lesbians in our study (Fig. 1A) conform with this notion that differences in pre- natal androgen exposure might account for some sexual minorities among women, but not among men (Fig. 1B).

Regardless of sexual orientation, men showed unprecedented decreases in testosterone following the TSST. Studies contrasting time changes in testosterone following the TSST report no differences at $+10 \mathrm{~min}$ or $+25 \mathrm{~min}$ (Schoofs and Wolf, 2011), but then increased testosterone was detected at $+30 \mathrm{~min}$ (Gerra et al., 2000), at $+40 \mathrm{~min}$ (Lennartsson et al., 2012a), and finally throughout personalized peak levels ranging from $+25 \mathrm{~min},+47 \mathrm{~min}$, and $+77 \mathrm{~min}$ (Bedgood et al., 2014). Our finding of decreased testosterone from -15 min pre-TSST to +15 min post-TSST is surprisingly fast. One possibility is rapid autonomic regulation and/or anticipatory modulation (e.g., visit order) of stress response signalling that dampens testosterone concentrations for men. Despite no clear evidence that luteinizing hormone differs among LGB individuals, luteinizing releasing hormone secretion is regulated by hypothalamic neurons that are modulated by fast-acting catecholamines (Gooren et al., 1990). Alternatively, the main methodological difference between previous HPG-axis TSST studies and our study is that we used the 'panel out' TSST variation where the evaluative judge is hidden behind a one-way mirror. Although purely speculative, the lack of visual social-evaluative threat might decrease testosterone among men and could be explored further in the stress reactivity literature.

Given that sex hormones are characterized by situationaldependent release, and are synergized by other biobehavioral functions (Newman and Josephs, 2009; Turan et al., 2014), constitutional factors and social behaviors might modulate sex hormone physiology. For instance, testosterone and cortisol interact to predict aggressive and dominant behaviors (Carré and Mehta, 2011). High levels of sexual minority stress coupled with inadequate coping resources amongst lesbian and bisexual women (IOM, 2011) may recalibrate HPG axis equilibria to favour an endocrine milieu underlying alternative coping strategies. Future studies must assess these psychosocial constructs in better detail than the current study allowed. By contrast, the absolute absence of differences in sex hormones among men is remarkably consistent with existing literature (Meyer-Bahlburg, 1977).

While males who identify with a sexual minority identity/orientation face similar pressures and stigma, there is empirical and theoretical justifications to consider their coping mechanisms separate from sexual minority women (Lewis et al., 2012). Considering this framework, a lesbian/bisexual specific up-regulation of testosterone as a result of a unique combination of psychoso- 
cial stressors is a possible mechanism. However, this relationship is complicated by reports of bisexual women in comparison to lesbian women experiencing more adverse mental health outcomes as a result of maladaptive coping mechanisms (Lehavot, 2012). Given the skewed nature of our sample towards bisexual women $(n=13)$ as opposed to lesbian women $(n=8)$, the former group may be driving effects.

\subsection{Strengths and limitations}

This study is primarily limited by a small self-selected sample with restricted generalizability. In the context of our nonprobability sample, our results may also not apply universally to international LGB communities. This selection bias is noteworthy given Montreal's liberal socio-cultural climate that may not generalize to other geopolitical strata. Moreover, combining our homosexual and bisexual sub-groups to increase power may have obscured important nuances among LGB communities and their unique psychosocial experiences. Given that $62 \%$ of our sample's sexual minority women were bisexual, the current findings may be more specific to bisexual women. Bisexuals are believed to experience the highest risk of psychopathology compared to other sexual orientation sub-groups (Bostwick et al., 2010; Persson et al., 2014). By the same logic, very few bisexual men participated, rendering our results more applicable to gay men comprising $80 \%$ of our sample's sexual minority men. Finally, we did not test transgender individuals that ought to be included in future studies.

In terms of methodology, it is important to note that salivary analytes like testosterone are very sensitive to treatment conditions (Granger et al., 2004) and bioactive fractions are often camouflaged by biochemical confounders. For instance, previous studies have urged researchers to assess how estrogen-androgen imbalances might be associated to binding globulins (Jaffee et al., 1980 ) that we did not account for in the current study. Moreover, salivary sex hormone concentrations represent bioactive "free" fractions that many consider more reliable; however, it is important to note that earlier research assessing sexual orientation and HPG-axis differences that used free concentrations from plasma were in contradiction to each other (Doerr et al., 1976; Stahl et al., 1976). That we would find that serum DHEA-S was higher in lesbian/bisexual women compared to heterosexual women complements similar directions of findings for salivary testosterone and progesterone. Ideally, we should have measured sex hormones in blood as well to address this issue; however, it is important to emphasize that the supplemental analysis of individual biomarkers among women was executed as a post-hoc exploration.

Beyond identified methodological differences that limit study comparisons according to extraction from either plasma, serum, urine, and/or saliva (Meyer-Bahlburg, 1977), other factors beyond those assessed in our study could further modulate HPG-axis activities. Lastly, the lack of basal endocrine differences according to menstrual status speak to the challenge of self-reports (Bachand et al., 2009; Jukic et al., 2008; Small et al., 2007) that do not consistently correspond with endocrine concentrations presumed to vary according to diverse reproductive statuses. Future studies will need to take special attention to address this issue with more refined control of reproductive functions among both sexes (Juster et al., 2016b).

Our study nevertheless has notable strengths that address several key recommendations voiced in Meyer-Bahlburg's reviews of the 1970s literature. Perhaps most importantly, the current study is to our knowledge the first to address psychosocial stress directly in relation to HPG-axis functioning among people of diverse sexual orientations. Nevertheless, our results can only be considered preliminary and will need to be replicated in future studies that can provide more complete psychophysiological profiles to eluci- date potential mechanisms involved. Like the majority of studies in this area, our cross-sectional design precludes any insights into the causes and consequences of these HPG-axis profiles that are moreover independent of confounders such as menstrual status, oral contraceptive use, visit order, or age. We therefore confirm that extraneous stress-related factors help explain particularities and inconsistencies observed in this mixed literature (Kolodny et al., 1972; Meyer-Bahlburg, 1979).

\subsection{Conclusions}

To summarize, sex hormones vary according to sexual orientation in women, but also importantly according to stress indices. Lesbian/bisexual women showed elevated basal testosterone and progesterone concentrations, while no differences were found among gay/bisexual men in comparison to sex-matched heterosexual controls. In addition, lesbian/bisexual women showed higher concentrations of the androgen precursor DHEA-S as well as LDL-cholesterol. Stress indices included cortisol systemic output, allostatic load indexing multi-systemic dysregulations, and perceived stress were variously correlated to sex hormones, confirming that stress processes should be accounted for in studies of sex hormones and vice-versa (Juster et al., 2016b; Stephens et al., 2016; Viau, 2002). These complex inter-individual differences in HPG-axis concentrations are likely the product of unique sociocultural factors like stigma rather than strictly explained by sexual orientation.

\section{Contributions}

R.P.J., S.J.L., and J.C.P. designed this study. R.P.J. collected all data. R.P.J., S.J.L., J.P.C., A.M., J.G.P., and C.C. assisted with statistical analyses and interpretations of the data. R.P.J., D.A., C.R., and P.J.J. helped with manuscript writing and editing.

\section{Conflict of interest}

The authors unanimously declare no conflicts of interest.

\section{Role of the funding source}

This study was funded by a grant (222055) from the Canadian Institutes of Health Research to S.J.L. who held a Senior Investigator Chair on Gender and Mental Health from the Canadian Institute of Gender and Health (GSC 91039). R.P.J. held a Doctoral scholarship from the Institute of Aging of the Canadian Institutes of Health Research (SIA 95402).

\section{Acknowledgements}

We are very grateful to Helen Findlay for performing salivary biochemical assays with such marvellous meticulousness. Thanks are also expressed to the nurses Louise Normandeau, Soaud Lahlafi, and Carole Feltrin for blood extraction as well as Edouard Kouassi for biochemical assays. We wish to thank the two anonymous reviewers for their helpful comments as well as Anke Ehrhardt and Heino Meyer-Bahlburg for their inspirational guidance. Finally, we credit the two-man Internet music group Threebrain for their 2001 flash video that inspired our title.

\section{References}

Andrews, J., Wadiwalla, M., Juster, R.P., Lord, C., Lupien, S.J., Pruessner, J.C., 2007. Effects of manipulating the amount of social-evaluative threat on the cortisol stress response in young healthy men. Behav. Neurosci. 121, 871-876.

Bachand, A.M., Cragin, L.A., Reif, J.S., 2009. Reliability of retrospectively assessed categorical menstrual cycle length data. Ann. Epidemiol. 19, 501-503. 
Banks, A., Gartrell, N.K., 1995. Hormones and sexual orientation: a questionable link. J. Homosex. 28, 247-268.

Barlow, D.H., Abel, G.G., Blanchard, E.B., Mavissakalian, M., 1974. Plasma testosterone levels and male homosexuality: a failure to replicate. Arch. Sex. Behav. 3, 571-575.

Bedgood, D., Boggiano, M.M., Turan, B., 2014. Testosterone and social evaluative stress: the moderating role of basal cortisol. Psychoneuroendocrinology 47 107-115.

Birk, L., Williams, G.H., Chasin, M., Rose, L.I., 1973. Serum testosterone levels in homosexual men. N. Engl. J. Med. 289, 1236-1238.

Bostwick, W.B., Boyd, C.J., Hughes, T.L., McCabe, S.E., 2010. Dimensions of sexual orientation and the prevalence of mood and anxiety disorders in the United States. Am. J. Public Health 100, 468-475.

Brodie, H.K., Gartrell, N., Doering, C., Rhue, T., 1974. Plasma testosterone levels in heterosexual and homosexual men. Am. J. Psychiatry 131, 82-83.

Carré, J.M., Mehta, P.H., 2011. Importance of considering testosterone-cortiso interactions in predicting human aggression and dominance. Aggress. Behav. 37, 489-491.

Cohen, S., Kamarck, T., Mermelstein, R., 1983. A global measure of perceived stress. J. Health Soc. Behav. 24, 385-396.

Dickerson, S.S., Kemeny, M.E., 2004. Acute stressors and cortisol responses: a theoretical integration and synthesis of laboratory research. Psychol. Bull. 130, 355-391.

Doerr, P., Kockott, G., Vogt, H.J., Pirke, K.M., Dittmar, F., 1973. Plasma testosterone, estradiol, and semen analysis in male homosexuals. Arch. Gen. Psychiatry 29, 829-833.

Doerr, P., Pirke, K.M., Kockott, G., Dittmar, F., 1976. Further studies on sex hormones in male homosexuals. Arch. Gen. Psychiatry 33, 611-614.

Downey, J., Ehrhardt, A.A., Schiffman, M., Dyrenfurth, I., Becker, J., 1987. Sex hormones in lesbian and heterosexual women. Horm. Behav. 21, 347-357.

Ehrhardt, A.A., Meyer-Bahlburg, H.F., 1981. Effects of prenatal sex hormones on gender-related behavior. Science 211, 1312-1318.

Ehrhardt, A.A., Meyer-Bahlburg, H.F., Rosen, L.R., Feldman, J.F., Veridiano, N.P., Zimmerman, I., McEwen, B.S., 1985. Sexual orientation after prenatal exposure to exogenous estrogen. Arch. Sex. Behav. 14, 57-77.

Ellis, L., Ames, M.A., 1987. Neurohormonal functioning and sexual orientation: a theory of homosexuality-heterosexuality. Psychol. Bull. 101, 233-258.

Evans, R.B., 1972. Physical and biochemical characteristics of homosexual men. J. Consult. Clin. Psychol. 39, 140-147.

Fleischman, D.S., Fessler, D.M., Cholakians, A.E., 2015. Testing the affiliation hypothesis of homoerotic motivation in humans: the effects of progesterone and priming. Arch. Sex. Behav. 44, 1395-1404.

Friedman, R.C., Downey, J.I., 1994. Homosexuality. N. Engl. J. Med. 331, 923-930.

Friedman, R.C., Frantz, A.G., 1977. Plasma prolactin levels in male homosexuals. Horm. Behav. 9, 19-22

Fritz, C.O., Morris, P.E., Richler, J.J., 2012. Effect size estimates: current use, calculations, and interpretation. J. Exp. Psychol. Gen. 141, 2-18.

Gerra, G., Zaimovic, A., Zambelli, U., Timpano, M., Reali, N., Bernasconi, S. Brambilla, F., 2000. Neuroendocrine responses to psychological stress in adolescents with anxiety disorder. Neuropsychobiology 42, 82-92.

Gooren, L., Fliers, E., Courtney, D.O., 1990. Biological determinants of sexual orientation. Annu. Rev. Sex Res. 1, 175-196.

Granger, D.A., Shirtcliff, E.A., Booth, A., Kivlighan, K.T., Schwartz, E.B., 2004. The trouble with salivary testosterone. Psychoneuroendocrinology 29, 1229-1240.

Gray, P.B., Campbell, B.C., Marlowe, F.W., Lipson, S.F., Ellison, P.T., 2004. Socia variables predict between-subject but not day-to-day variation in the testosterone of US men. Psychoneuroendocrinology 29, 1153-1162.

Griffiths, P.D., Merry, J., Browning, M.C., Eisinger, A.J., Huntsman, R.G., Lord, E.J., Polani, P.E., Tanner, J.M., Whitehouse, R.H., 1974. Homosexual women: an endocrine and psychological study. J. Endocrinol. 63, 549-556.

Grimbos, T., Dawood, K., Burriss, R.P., Zucker, K.J., Puts, D.A., 2010. Sexual orientation and the second to fourth finger length ratio: a meta-analysis in men and women. Behav. Neurosci. 124, 278-287.

Hatzenbuehler, M.L., Jun, H.J., Corliss, H.L., Austin, S.B., 2013. Structural stigma and cigarette smoking in a prospective cohort study of sexual minority and heterosexual youth. Ann. Behav. Med.

Hines, M., 2011. Prenatal endocrine influences on sexual orientation and on sexually differentiated childhood behavior. Front. Neuroendocrinol. 32 170-182.

IOM, 2011. The Health of Lesbian, Gay, Bisexual, and Transgender People: Building a Foundation for Better Understanding. The National Academies Press, Washinton, DC.

Jaffee, W.L., McCormack, W.M., Vaitukaitis, J.L., 1980. Plasma hormones and the sexual preferences of men. Psychoneuroendocrinology 5, 33-38.

Jukic, A.M., Weinberg, C.R., Wilcox, A.J., McConnaughey, D.R., Hornsby, P., Baird, D.D., 2008. Accuracy of reporting of menstrual cycle length. Am. J. Epidemiol. 167, 25-33.

Juster, R.P., Smith, N.G., Ouellet, E., Sindi, S., Lupien, S.J., 2013a. Sexual orientation and disclosure in relation to psychiatric symptoms, diurnal cortisol, and allostatic load. Psychosom. Med. 75, 103-116.

Juster, R.P., Smith, N.G., Ouellet, E., Sindi, S., Lupien, S.J., 2013b. Sexual orientation and disclosure in relation to psychiatric symptoms, diurnal cortisol, and allostatic load. Psychosom. Med. 75, 103-116.

Juster, R.P., Hatzenbuehler, M.L., Mendrek, A., Pfaus, J.G., Smith, N.G., Johnson, P.J., Lefebvre-Louis, J.P., Raymond, C., Marin, M.F., Sindi, S., Lupien, S.J., Pruessner,
J.C., 2015. Sexual orientation modulates endocrine stress reactivity. Biol. Psychiatry 77, 668-676.

Juster, R.P., Raymond, C., Desrochers, A.B., Bourdon, O., Durand, N., Wan, N., Pruessner, J.C., Lupien, S.J., 2016a. Sex hormones adjust sex-specific reactive and diurnal cortisol profiles. Psychoneuroendocrinology 63, 282-290.

Juster, R.P., Ouellet, E., Lefebvre-Louis, J.P., Sindi, S., Johnson, P.J., Smith, N.G., Lupien, S.J., 2016b. Retrospective coping strategies during sexual identity formation and current biopsychosocial stress. Anxiety Stress Coping 29, $119-138$.

Kirschbaum, C., Wüst, S., Hellhammer, D.H., 1992. Consistent sex differences in cortisol responses to psychological stress. Psychosom. Med. 54, 648-657.

Kirschbaum, C., Pirke, K.M., Hellhammer, D.H., 1993. The 'Trier Social Stress Test'-a tool for investigating psychobiological stress responses in a laboratory setting. Neuropsychobiology 28, 76-81.

Kirschbaum, C., Pirke, K.M., Hellhammer, D.H., 1995. Preliminary evidence fo reduced cortisol responsivity to psychological stress in women using ora contraceptive medication. Psychoneuroendocrinology 20, 509-514.

Kirschbaum, C., Kudielka, B.M., Gaab, J., Schommer, N.C., Hellhammer, D.H., 1999. Impact of gender, menstrual cycle phase, and oral contraceptives on the activity of the hypothalamus-pituitary-adrenal axis. Psychosom. Med. 61, 154-162.

Klein, F., Sepekoff, B., Wolf, T.J., 1990. Sexual orientation: a multivariable dynamic process. In: Geller, T. (Ed.), Bisexuality: A Reader and Sourcebook. Times Change Press.

Kolodny, R.C., Masters, W.H., Hendryx, J., Toro, G., 1971. Plasma testosterone and semen analysis in male homosexuals. N. Engl. J. Med. 285, 1170-1174.

Kolodny, R.C., Jacobs, L.S., Masters, W.H., Toro, G., Daughaday, W.H., 1972. Plasma gonadotrophins and prolactin in male homosexuals. Lancet 2, 18-20.

Kreuz, L.E., Rose, R.M., Jennings, J.R., 1972. Suppression of plasma testosterone levels and psychological stress: a longitudinal study of young men in officer candidate school. Arch. Gen. Psychiatry 26, 479-482.

Lehavot, K., 2012. Coping strategies and health in a national sample of sexual minority women. Am. J. Orthopsychiatry 82, 494-504.

Lennartsson, A.K., Kushnir, M.M., Bergquist, J., Billig, H., Jonsdottir, I.H., 2012a. Sex steroid levels temporarily increase in response to acute psychosocial stress in healthy men and women. Int. J. Psychophysiol. 84, 246-253.

Lennartsson, A.K., Kushnir, M.M., Bergquist, J., Jonsdottir, I.H., 2012b. DHEA and DHEA-S response to acute psychosocial stress in healthy men and women. Biol. Psychol. 90, 143-149.

Lewis, R., Kholodkov, T., Derlega, V.J., 2012. Still stressful after all these years: a review of lesbians' and bisexual women's minority. J. Lesbian Stud. 16, 30-44.

Loraine, J.A., Ismail, A.A., Adamopoulos, D.A., Dove, G.A., 1970. Endocrine function in male and female homosexuals. Br. Med. J. 4, 406-409.

Loraine, J.A., Adamopoulos, D.A., Kirkham, K.E., Ismail, A.A., Dove, G.A., 1971. Patterns of hormone excretion in male and female homosexuals. Nature 234 552-555.

Manning, J.T., 2002. Digit Ratio: A Pointer to Fertility, Behaviour, and Health Rutgers. University Press, Piscataway, NJ.

Marin, M.F., Morin-Major, J.K., Schramek, T.E., Beaupre, A., Perna, A., Juster, R.P., Lupien, S.J., 2012. There is no news like bad news: women are more remembering and stress reactive after reading real negative news than men. PLoS One 7, e47189.

Mason, J.W. 1968. A review of psychoendocrine research on the sympathetic-adrenal medullary system. Psychosom. Med. 30 (Suppl), 631-653.

McEwen, B.S., Stellar, E., 1993. Stress and the individual: mechanisms leading to disease. Arch. Intern. Med. 153, 2093-2101.

McFadden, D., Pasanen, E.G. 1998. Comparison of the auditory systems of heterosexuals and homosexuals: click-evoked otoacoustic emissions. Proc. Natl. Acad. Sci. U. S. A. 95, 2709-2713.

McFadden, D., 2002. Masculinization effects in the auditory system. Arch. Sex. Behav. 31, 99-111.

Meyer, I.H., 2003. Prejudice, social stress, and mental health in lesbian, gay, and bisexual populations: conceptual issues and research evidence. Psychol. Bull. 129, 674-697.

Meyer-Bahlburg, H.F., Ehrhardt, A.A., Rosen, L.R., Gruen, R.S., Veridiano, N.P., Vann, F.H., Neuwalder, H.F., 1995. Prenatal estrogens and the development of homosexual orientation. Dev. Psychol. 31, 12-21.

Meyer-Bahlburg, H.F., 1977. Sex hormones and male homosexuality in comparative perspective. Arch. Sex. Behav. 6, 297-325.

Meyer-Bahlburg, H.F., 1979. Sex hormones and female homosexuality: a critical examination. Arch. Sex. Behav. 8, 101-119.

Newman, M.L., Josephs, R.A., 2009. Testosterone as a personality variable. J. Res. Personal. 43, 258-259.

Parks, G.A., Korth-Schutz, S., Penny, R., Hilding, R.F., Dumars, K.W., Frasier, S.D., New, M.I., 1974. Variation in pituitary-gonadal function in adolescent male homosexuals and heterosexuals. J. Clin. Endocrinol. Metab. 39, 796-801.

Persson, T.J., Pfaus, J.G., Ryder, A.G., 2014. Explaining mental health disparities for non-monosexual women: abuse history and risky sex, or the burdens of non-disclosure? Soc. Sci. Med.

Phoenix, C.H., Goy, R.W., Gerall, A.A., Young W.C., 1959. Organizing action of prenatally administered testosterone propionate on the tissues mediating mating behavior in the female guinea pig. Endocrinology 65, 369-382.

Pillard, R.C., Rose, R.M., Sherwood, M., 1974. Plasma testosterone levels in homosexual men. Arch. Sex. Behav. 3, 453-458.

Pruessner, J.C., Kirschbaum, C., Meinlschmid, G., Hellhammer, D.H., 2003. Two formulas for computation of the area under the curve represent measures of 
total hormone concentration versus time-dependent change. Psychoneuroendocrinology 28, 916-931.

Rohde, W., Stahl, F., Dorner, G., 1977. Plasma basal levels of FSH, LH and testosterone in homosexual men. Endokrinologie 70, 241-248.

Schoofs, D., Wolf, O.T., 2011. Are salivary gonadal steroid concentrations influenced by acute psychosocial stress? A study using the Trier Social Stress Test (TSST). Int. J. Psychophysiol. 80, 36-43.

Seeman, E., Singer, B.H., Rowe, J., Horwitz, R.I., McEwen, B., 1997. Price of adaptation-allostatic load and its health consequences. Arch. Intern. Med. $157,2259-2268$

Seyler Jr., L.E., Canalis, E., Spare, S., Reichlin, S., 1978. Abnormal gonadotropin secretory responses to LRH in transsexual women after diethylstilbestrol priming. J. Clin. Endocrinol. Metab. 47, 176-183.

Sindi, S., Fiocco, A.J., Juster, R.P., Pruessner, J., Lupien, S.J., 2013. When we test, do we stress? Impact of the testing environment on cortisol secretion and memory performance in older adults. Psychoneuroendocrinology 38 , 1388-1396.

Small, C.M., Manatunga, A.K., Marcus, M., 2007. Validity of self-reported menstrual cycle length. Ann. Epidemiol. 17, 163-170.

Stahl, F., Dörmer, G., Wood, G., Paczynski, M., 1976. Significantly decreased apparently free testosterone levels in plasma of male homosexuals. Endokrinologies 68, 115-117.
Stephens, M.A.C. Mahon, P.B., McCaul, M.E., Wand, G.S., 2016

Hypothalamic-pituitary-adrenal axis response to acute psychosocial stress: effects of biological sex and circulating sex hormones.

Psychoneuroendocrinology 66, 47-55.

Tourney, G., Hatfield, L.M., 1973. Androgen metabolism in schizophrenics, homosexuals, and normal controls. Biol. Psychiatry 6, 23-36.

Turan, B., Guo, J., Boggiano, M.M., Bedgood, D., 2014. Dominant, cold, avoidant, and lonely: basal testosterone as a biological marker for an interpersonal style. J. Res. Personal. 50, 84-89.

Viau, V., 2002. Functional cross-talk between the hypothalamic-pituitary-gonadal and -adrenal axes. J. Neuroendocrinol. 14, 506-513.

Wadiwalla, M., Andrews, J., Lai, B., Buss, C., Lupien, S.J., Pruessner, J.C., 2010. Effects of manipulating the amount of social-evaluative threat on the cortisol stress response in young healthy women. Stress 13, 214-220.

Zuloaga, D.G., Puts, D.A., Jordan, C.L., Breedlove, S.M., 2008. The role of androgen receptors in the masculinization of brain and behavior: what we've learned from the testicular feminization mutation. Horm. Behav. 53, 613-626.

van Anders, S.M., Hampson, E., Watson, N.V., 2006. Seasonality, waist-to-hip ratio, and salivary testosterone. Psychoneuroendocrinology 31, 895-899. 